\title{
Dynamics of an SIR Epidemic Model with Information Variable and Limited Medical Resources Revisited
}

\author{
Caijuan Yan, ${ }^{1}$ Jianwen Jia, ${ }^{1}$ and Zhen Jin ${ }^{2}$ \\ ${ }^{1}$ School of Mathematics and Computer Science, Shanxi Normal University, Shanxi, Linfen 041004, China \\ ${ }^{2}$ Department of Mathematics, North University of China, Shanxi, Taiyuan 030051, China
}

Correspondence should be addressed to Jianwen Jia; jiajw.2008@163.com

Received 11 December 2013; Accepted 20 February 2014; Published 27 April 2014

Academic Editor: Guang Zhang

Copyright (C) 2014 Caijuan Yan et al. This is an open access article distributed under the Creative Commons Attribution License, which permits unrestricted use, distribution, and reproduction in any medium, provided the original work is properly cited.

The stability of the SIR epidemic model with information variable and limited medical resources was studied. When the basic reproduction ratio $\mathscr{R}_{0}<1$, there exists the disease-free equilibrium and when the basic reproduction ratio $\mathscr{R}_{0}>1$, we obtain the sufficient conditions of the existence of the endemic equilibrium. The local asymptotical stability of equilibrium is verified by analyzing the eigenvalues and using the Routh-Hurwitz criterion. We also discuss the global asymptotical stability of the endemic equilibrium by autonomous convergence theorem. A numerical analysis is given to show the effectiveness of the main results.

\section{Introduction}

Mathematical epidemiology, that is, the building and analysis of mathematical models describing the spread and control of infectious diseases, plays an important role in the areas of biology. Various epidemic models have been proposed and explored extensively and great progress has been achieved in the studies of disease control and prevention. In the classical epidemic models, it is usually assumed that the change of the total population size satisfies logistic model or constant. We used nonlinear incidence, standard incidence rate, general incidence rate, and saturated incidence rate to concentrate on describing the spread of disease through the population. The analysis of all of these models has also been done by some other workers; see for example $[1,2]$ and the references therein.

In order to control the spread of epidemic, we consider the new variable $Z$, called information variable which summarizes information about the current state of the disease that is depending on current values of state variables and also summarizes information about past values of state variables. Many authors have used this variable in their models (see, e.g., [3-5]).

In many models, it is usually assumed that the removal rate of the infective is proportional to the number of the infective, which implies that the medical resources such as drugs, vaccines, hospital beds, and isolation places are very sufficient for the infectious disease. However, in reality, every country has an appropriate or limited capacity for treatment. Recently, Kar and Mondal have introduced the continually differentiable treatment function $h(I)=r I /(1+\alpha I)$ (see in [6]), where $r / \alpha$ models the maximal supply of available medical resources per unit time and $1 /(1+\alpha I)$ describes the reverse effect of the infected individuals being delayed for treatment, which have important effects on the spread of infectious disease. $r / \alpha$ and the efficiency of the supply of available medical resources $1 /(1+\alpha I)$ are independent. In many developed countries, in addition to the limitedness of the medical resources, the efficiency of the supply of available medical resources also has an important effect on the transmission of infectious disease, which depends on many factors such as the control strategies and the production of drugs or vaccines. In [7], the SIR models with limited medical resources have been studied.

To better understand their effects on the spread of infectious diseases, in this paper, we will discuss the model with saturated incidence rate $\beta S I /(1+\alpha I)$ (see [7]), information variable

$$
Z(t)=\int_{-\infty}^{t} S \frac{1}{T} \exp \left(-\frac{1}{T}(t-\tau)\right) \mathrm{d} \tau,
$$

and treatment function $h(I)=b I /(\omega+I)$. 
The paper is organized as follows: in Section 2, we explore the existence of disease-free equilibria point and endemic equilibrium point and investigate the effect of the limited medical resources and their supply efficiency; in Section 3, we analyze the local asymptotic stability of the diseasefree equilibria and the endemic equilibrium; in Section 4, we analyze the global asymptotic stability of the endemic equilibrium and present a global analysis of the model; a numerical analysis and a simple discussion are given to conclude this paper in Section 5.

\section{The Model and the Existence of Equilibrium}

In paper [6], a simple example is the classical SIR epidemic model with information variable and saturated incidence rate which has been studied as follows:

$$
\begin{gathered}
S^{\prime}(t)=r S\left(1-\frac{S}{k}\right)-\frac{\beta S I}{1+\alpha S}, \\
I^{\prime}(t)=\frac{\beta I Z}{1+\alpha Z}-\left(\mu_{1}+\gamma\right) I, \\
Z(t)=\int_{-\infty}^{t} S \frac{1}{T} \exp \left(-\frac{1}{T}(t-\tau)\right) \mathrm{d} \tau, \\
R^{\prime}(t)=\gamma I-\mu_{2} R .
\end{gathered}
$$

In paper [7], another simple example is the classical SIR epidemic model with limited medical resources which carefully investigated the dynamics of the following SIR model:

$$
\begin{gathered}
S^{\prime}(t)=\Lambda-\frac{\beta S I}{1+k I}, \\
I^{\prime}(t)=\frac{\beta S I}{1+k I}-(d+\gamma+\varepsilon) I-\frac{\alpha I}{\omega+I}, \\
R^{\prime}(t)=\gamma I-d R+\frac{\alpha I}{\omega+I} .
\end{gathered}
$$

Based on the above motivations, in this paper, we further explore the SIR epidemic model with saturated incidence rate $\beta S I /(1+\alpha I)$, information variable $Z(t)$, and a continually differentiable treatment function (see [8]) $h(I)=b I /(\omega+I)$ to characterize the saturation phenomenon of the limited medical resources. The model can be described by the following system of equations:

$$
\begin{gathered}
S^{\prime}(t)=r S\left(1-\frac{S}{k}\right)-\frac{\beta S I}{1+\alpha I}, \\
I^{\prime}(t)=\frac{\beta I Z}{1+\alpha Z}-\left(\mu_{1}+\gamma+\varepsilon\right) I-\frac{b I}{\omega+I}, \\
Z(t)=\int_{-\infty}^{t} S \frac{1}{T} \exp \left(-\frac{1}{T}(t-\tau)\right) \mathrm{d} \tau, \\
R^{\prime}(t)=\gamma I-\mu_{2} R+\frac{b I}{\omega+I},
\end{gathered}
$$

where $S(t), I(t)$, and $R(t)>0$ and $S(t), I(t)$, and $R(t)$ denote the numbers of susceptible, infective, and recovered individuals at time $t$, respectively. $r$ is the intrinsic growth rate of susceptibles, $k$ is the carrying capacity of susceptibles, $\alpha$ is the saturation factor that measures the inhibitory effect, $\beta$ is the transmission or contact rate, $\mu_{1}, \mu_{2}$ are the natural death rate of the infective and recovered individuals, $\gamma$ is the natural recovery rate, $\varepsilon$ is the disease-related mortality, $b \geq 0$ is the maximal medical resources supplied per unit time, and $\omega>0$ is half-saturation constant. $r, b, \mu_{1}, \mu_{2}, \gamma, \alpha, \beta, k, \omega$ are all positive.

Then the nonlinear integrodifferential system (4) can be transformed into the following set of nonlinear ordinary differential questions:

$$
\begin{gathered}
S^{\prime}(t)=r S\left(1-\frac{S}{k}\right)-\frac{\beta S I}{1+\alpha I}, \\
I^{\prime}(t)=\frac{\beta I Z}{1+\alpha Z}-\left(\mu_{1}+\gamma+\varepsilon\right) I-\frac{b I}{\omega+I}, \\
Z^{\prime}(t)=\frac{1}{T}(S-Z), \\
R^{\prime}(t)=\gamma I-\mu_{2} R+\frac{b I}{\omega+I} .
\end{gathered}
$$

Since the dynamical behavior of the last equation of system (5), that is, the dynamics of $R$, depends only on the dynamics of $I$, the dynamics of $R$ is the same as $I$. So we do not consider the last equation of system (5) in our discussion. Here we will study the following nonlinear ordination differential equations:

$$
\begin{gathered}
S^{\prime}(t)=r S\left(1-\frac{S}{k}\right)-\frac{\beta S I}{1+\alpha S}, \\
I^{\prime}(t)=\frac{\beta I Z}{1+\alpha S}-\left(\mu_{1}+\gamma+\varepsilon\right) I-\frac{b I}{\omega+I}, \\
Z^{\prime}(t)=\frac{1}{T}(S-Z) .
\end{gathered}
$$

Denote $\mathscr{R}_{0}=\beta k /\left(\mu_{1}+\gamma+\varepsilon+(b / \omega)\right)(1+k \alpha), \overline{\mathscr{R}}_{0}=$ $\beta k /\left(\mu_{1}+\gamma+\varepsilon\right)(1+k \alpha)$.

Theorem 1. (1) The system (6) has a trivial equilibrium $E_{0}=$ $(0,0,0)$ and the disease-free equilibrium $E_{1}=(k, 0, k)$.

(2) If $\mathscr{R}_{0}>1$, further if $\overline{\mathscr{R}}_{0}<1-(k \beta / r(1+k \alpha))$, the system (6) has one endemic equilibrium $E^{*}=\left(S^{*}, I^{*}, Z^{*}\right)$ except the disease-free equilibrium.

Proof. (1) Let $I=0$, we have $S=Z=0$ or $S=Z=k$; it is not easy to find that the system has a trivial equilibrium and the disease-free equilibrium $E_{0}=(0,0,0)$ and $E_{1}=(k, 0, k)$.

(2) If $\mathscr{R}_{0}>1$, from the first question of (6), we have $S^{*}=$ $(k / r)\left(r-\left(\beta I^{*} /\left(1+\alpha I^{*}\right)\right)\right)$, from the third question of (6), we also have $Z^{*}=S^{*}$. Then substituting them into the first question of (6) yields

$$
A I^{2}+B I+C=0
$$


where

$$
\begin{aligned}
A= & k \alpha \beta-\frac{k \beta^{2}}{r}-\left(\mu_{1}+\gamma+\varepsilon\right) \\
& \times\left[(1+k \alpha) \alpha-\frac{k \alpha \beta}{r}\right] \\
= & k \beta\left\{\alpha-\frac{\beta}{r}-\frac{1}{\overline{\mathscr{R}}_{0}}\left[\alpha-\frac{k \alpha \beta}{r(1+k \alpha)}\right]\right\}, \\
C= & \beta k \omega-\left(\mu_{1}+\gamma+\varepsilon\right) \omega(1+k \alpha)-b(1+k \alpha) \\
= & \omega\left(\mu_{1}+\gamma+\varepsilon+\frac{b}{\omega}\right)(1+k \alpha)\left(\mathscr{R}_{0}-1\right) .
\end{aligned}
$$

It is clear that if $\mathscr{R}_{0}>1$ and $\overline{\mathscr{R}}_{0}<1-(k \beta / r(1+k \alpha))$, then $1<\mathscr{R}_{0}<\overline{\mathscr{R}}_{0}<1-(k \beta / r(1+k \alpha))$. We have $A<0, C>0$.
The roots of (7) $I_{1}, I_{2}$ satisfy

$$
I_{1} \cdot I_{2}=\frac{C}{A}<0 .
$$

Therefore, if $\mathscr{R}_{0}>1$ and $\overline{\mathscr{R}}_{0}<1-(k \beta / r(1+k \alpha))$, it is obvious that the sign of $B$ is positive or negative. There always exists a unique positive root, that is, $I^{*}$. So (6) has one endemic equilibrium.

\section{The Local Stability Analysis of Equilibria and Bifurcation}

In this section, we will examine the local stability of the equilibria by analyzing the eigenvalues of the Jacobian matrices of (6) at the equilibria and using Routh-Hurwitz criterion.

Let $\bar{E}=(\bar{S}, \bar{I}, \bar{R})$ be the arbitrarily equilibrium of the system (6); then the Jacobian matrix at $\bar{E}$ of the system (6) is

$$
J(\bar{S}, \bar{I}, \bar{Z})=\left(\begin{array}{ccc}
r\left(1-\frac{\bar{S}}{k}\right)-\frac{\beta \bar{I}}{1+\alpha \bar{I}}-\frac{r}{k} \bar{S} & \frac{-\beta \bar{S}}{1+\alpha \bar{I}} & 0 \\
0 & \frac{\beta \bar{Z}}{1+\alpha \bar{Z}}-\left(\mu_{1}+\gamma+\varepsilon\right)-\frac{b \omega}{(\omega+\bar{I})^{2}} & \frac{\beta \bar{I}}{(1+\alpha \bar{Z})^{2}} \\
\frac{1}{T} & 0 & -\frac{1}{T}
\end{array}\right)
$$

Theorem 2. The free equilibrium point $E_{0}=(0,0,0)$ is unstable for any $T>0$.

Proof. The Jacobian matrix at $E_{0}$ is

$$
J\left(E_{0}\right)=\left(\begin{array}{ccc}
r & 0 & 0 \\
0 & -\left(\mu_{1}+\gamma+\varepsilon\right)-\frac{b}{\omega} & 0 \\
\frac{1}{T} & 0 & -\frac{1}{T}
\end{array}\right) .
$$

The eigenvalues are $\lambda_{1}=r>0, \lambda_{2}=-\left(\mu_{1}+\gamma+\varepsilon\right)-(b / \omega)$, and $\lambda_{3}=-(1 / T)$. Therefore $E_{0}$ is unstable.

Theorem 3. (1) If $\mathscr{R}_{0}>1$, that is, $\beta k>\left(\mu_{1}+\gamma+\varepsilon+(b / \omega)\right)(1+$ $k \alpha)$, then $E_{1}=(k, 0, k)$ is unstable for all $T>0$.

(2) If $\mathscr{R}_{0}<1$, that is, $\beta k<\left(\mu_{1}+\gamma+\varepsilon+(b / \omega)\right)(1+k \alpha)$, then $E_{1}=(k, 0, k)$ is locally asymptotically stable for all $T>0$.

Proof. The Jacobian matrix at $E_{1}$ is

$$
J\left(E_{1}\right)=\left(\begin{array}{ccc}
-r & \beta k & 0 \\
0 & \frac{\beta k}{1+\alpha k}-\left(\mu_{1}+\gamma+\varepsilon+\frac{b}{\omega}\right) & 0 \\
\frac{1}{T} & 0 & -\frac{1}{T}
\end{array}\right) .
$$

The eigenvalues are $\lambda_{1}=-r, \lambda_{2}=\beta k /(1+\alpha k)-\left(\mu_{1}+\gamma+\varepsilon+\right.$ $(b / \omega))$, and $\lambda_{3}=-1 / T$.

(1) If $\mathscr{R}_{0}>1$, that is, $(\beta k /(1+\alpha k))>\mu_{1}+\gamma+\varepsilon+(b / \omega)$, then $\lambda_{2}>0$; therefore $E_{1}=(k, 0, k)$ is unstable.
(2) If $\mathscr{R}_{0}<1$, that is, $\beta k /(1+\alpha k)<\mu_{1}+\gamma+\varepsilon+$ $(b / \omega)$, then $\lambda_{2}<0$; therefore $E_{1}=(k, 0, k)$ is locally asymptotically stable.

Note. In Theorem 3, we can see that the stability of diseasefree equilibrium point $E_{1}$ changes from stable to unstable when $\mathscr{R}_{0}$ increases through 1 . Therefore, we use $\mathscr{R}_{0}$ as bifurcation parameter.

For simplicity, let

$$
\begin{aligned}
f_{W}(X, \beta, T) & =\left(\begin{array}{l}
f_{1}(X, \beta, T) \\
f_{2}(X, \beta, T) \\
f_{3}(X, \beta, T)
\end{array}\right) \\
& =\left(\begin{array}{c}
r S\left(1-\frac{S}{k}\right)-\frac{\beta S I}{1+\alpha I} \\
\frac{\beta I Z}{1+\alpha Z}-\left(\mu_{1}+\gamma+\varepsilon\right) I-\frac{b I}{\omega+I} \\
\frac{1}{T}(S-Z)
\end{array}\right)
\end{aligned}
$$

and $X=[S, I, Z]^{T}$.

Theorem 4. The system (6) undergoes transcritical bifurcation at the equilibrium point $E_{0}$ when bifurcation parameter $\mathscr{R}_{0}=$ 1. 
Proof. When $\mathscr{R}_{0}=1$, the Jacobian matrix at $E_{1}$ is

$$
\begin{aligned}
J\left(E_{1}\right) & =\left(\begin{array}{ccc}
-r & \beta k & 0 \\
0 & 0 & 0 \\
\frac{1}{T} & 0 & -\frac{1}{T}
\end{array}\right) \\
& =\left(\begin{array}{ccc}
-r & -\left(\mu_{1}+\gamma+\varepsilon+\frac{b}{\omega}\right) & 0 \\
0 & 0 & 0 \\
\frac{1}{T} & 0 & -\frac{1}{T}
\end{array}\right) .
\end{aligned}
$$

Then $J\left(E_{1}\right)$ has a geometrically simple zero eigenvalue with right eigenvector $\varphi=\left(1,-\left(r / \mu_{1}+\gamma+\varepsilon+(\alpha / \omega)\right), 1\right)^{T}$ and left eigenvector $\psi=(0,1,0)$.

Now

$$
\begin{gathered}
D_{\beta} f_{W}=\left(\begin{array}{c}
-\frac{S I}{1+\alpha I} \\
\frac{I Z}{1+\alpha Z} \\
0
\end{array}\right), \\
\left(\psi\left(D_{X} D_{\beta} f_{W}\right) \varphi\right)_{E_{1}} \\
=\left(\begin{array}{lll}
0 & 1 & 0
\end{array}\right) \\
\quad \times\left(\begin{array}{ccc}
-\frac{I}{1+\alpha I} & -\frac{S}{(1+\alpha I)^{2}} & 0 \\
0 & \frac{Z}{1+\alpha Z} & \frac{I}{(1+\alpha S)^{2}} \\
0 & 0 & 0
\end{array}\right)
\end{gathered}
$$

$$
\begin{aligned}
& J\left(E^{*}\right)=\left(\begin{array}{ccc}
r\left(1-\frac{S^{*}}{k}\right)-\frac{\beta I^{*}}{1+\alpha I^{*}}-\frac{r}{k} S^{*} & \frac{-\beta S^{*}}{\left(1+\alpha I^{*}\right)^{2}} & 0 \\
0 & \frac{\beta Z^{*}}{1+\alpha Z^{*}}-\left(\mu_{1}+\gamma+\varepsilon\right)-\frac{b \omega}{\left(\omega+I^{*}\right)^{2}} & \frac{\beta I^{*}}{\left(1+\alpha Z^{*}\right)^{2}} \\
\frac{1}{T} & 0 & -\frac{1}{T}
\end{array}\right) \\
& =\left(\begin{array}{ccc}
-\frac{r}{k} S^{*} & \frac{-\beta S^{*}}{\left(1+\alpha I^{*}\right)^{2}} & 0 \\
0 & \frac{b I^{*}}{\left(\omega+I^{*}\right)^{2}} & \frac{\beta I^{*}}{\left(1+\alpha Z^{*}\right)^{2}} \\
\frac{1}{T} & 0 & -\frac{1}{T}
\end{array}\right) .
\end{aligned}
$$

According to [9], the system (6) undergoes transcritical bifurcation at the disease-free equilibrium point $E_{1}$, hence the theorem.

Theorem 5. If $\mathscr{R}_{0}>1$ and $(r / k)>\alpha \beta I^{*} /\left(1+\alpha S^{*}\right)^{2}+$ $b I^{*} S^{*} /\left(\omega+I^{*}\right)^{2}$, then the endemic equilibrium $E^{*}$ is local asymptotical stability for $T<T_{*}$, where

$T_{*}$

$$
=\frac{-(Q-P+R /(Q-P))+\sqrt{[Q-P-R /(Q-P)]^{2}+4 P Q}}{2 P Q}
$$

$>0$.

Proof. The Jacobian matrix at $E^{*}$ is 


$$
\begin{aligned}
B & =\frac{1}{T} \frac{r}{k} S^{*}-\frac{1}{T} \frac{b I^{*}}{\left(\omega+I^{*}\right)^{2}}-\frac{r}{k} S^{*} \frac{b I^{*}}{\left(\omega+I^{*}\right)^{2}} \\
& =\frac{1}{T} P-\frac{1}{T} Q-P Q, \\
C= & -\frac{1}{T} \frac{r}{k} S^{*} \frac{b I^{*}}{\left(\omega+I^{*}\right)^{2}}+\frac{1}{T} \frac{\beta S^{*}}{\left(1+\alpha I^{*}\right)^{2}} \frac{\beta I^{*}}{\left(1+\alpha Z^{*}\right)^{2}} \\
= & -\frac{1}{T} P Q+\frac{1}{T} R .
\end{aligned}
$$

Let $P=(r / k) S^{*}, Q=b I^{*} /\left(\omega+I^{*}\right)^{2}$, and $R=$ $\left(\beta S^{*} /\left(1+\alpha I^{*}\right)^{2}\right)\left(\beta I^{*} /\left(1+\alpha Z^{*}\right)^{2}\right)$. We have

$$
A B-C=\frac{Q-P}{T^{2}}\left[(1+P T)(Q T-1)+\frac{R}{Q-P} T\right] .
$$

Let $f(T)=(1+P T)(Q T-1)+(R /(Q-P)) T$. Then $f(0)=$ -1 and $f(T)=0$ has an equine positive root:

$T_{*}$

$$
=\frac{-(Q-P+R /(Q-P))+\sqrt{[Q-P-R /(Q-P)]^{2}+4 P Q}}{2 P Q}
$$

$>0$.

Therefore, from the condition of Theorem 5 , we have $P>Q$, that is, $A>0$. If $T<T_{*}$, we have $f(T)<0$, that is, $A B-C>$ 0 . Hence, the theorem is trivially proved by Routh-Hurwitz criterion.

Note. If $T>T_{*}$, the equilibrium $E^{*}$ may be unstable (see Figure 3).

\section{The Global Stability Analysis of the Equilibrium Point}

Here we will shortly describe the general method by which the global stability analysis for the endemic equilibrium will be performed through the approach due to $\mathrm{Li}$ and Muldowney [10]. Consider the autonomous dynamical system:

$$
x^{\prime}=f(x),
$$

where $f: D \rightarrow R^{n}, D \subset R^{n}$ is an open set and simply connected and $x \in D, x \mapsto f(x) \in R^{n}, f(x) \in C^{1}(D)$.

Let $x^{*}$ be an equilibrium of (23). We recall that $x^{*}$ is said to be globally stable in $D$, if it is locally stable and all trajectories in $D$ converge to $x^{*}$. Assume that the following hypotheses hold.

$\left(H_{1}\right)$ There exists a compact absorbing set $K \subset D$.

$\left(H_{2}\right)$ Equation (23) has a unique equilibrium $x^{*}$ in $D$.
The basic idea of this method is that if the equilibrium $x^{*}$ is locally stable, then the stability is assured provided that $\left(H_{1}\right)$ and $\left(H_{2}\right)$ hold and no nonconstant periodic solution of (23) exists. Therefore, sufficient conditions on $f$ capable of precluding the existence of such solutions have to be detected.

$\mathrm{Li}$ and Muldowney showed that if $\left(H_{1}\right)$ and $\left(H_{2}\right)$ hold and (23) satisfies a Bendixson criterion, that is, robust under $C^{1}$ local $\epsilon$-perturbations of $f$ at all nonequilibrium nonwandering points for (23), then $x^{*}$ is globally stable robust under $C^{1}$ local $\epsilon$-perturbation and based on the introduced Lozinskil measure.

Let $P(x)$ be a $\left(\begin{array}{c}n \\ 2\end{array}\right) \times\left(\begin{array}{l}n \\ 2\end{array}\right)$ matrix-valued function, that is, $C^{1}$ on $D$, and consider

$$
B=P_{f} P^{-1}+P \frac{\partial f^{[2]}}{\partial x} P^{-1},
$$

where the matrix $P_{f}$ is

$$
\frac{\partial P_{i j}^{*}}{\partial x} f=\left.\frac{\mathrm{d} P_{i j}}{\mathrm{~d} t}\right|_{(23)}
$$

and the matrix $J^{[2]}$ is the second additive compound matrix of the Jacobian matrix $J$, that is, $J(x)=D f(x)$. Generally speaking, for a $n \times n$ matrix $J=\left(J_{i j}\right), J^{[2]}$ is a $\left(\begin{array}{c}n \\ 2\end{array}\right) \times\left(\begin{array}{c}n \\ 2\end{array}\right)$ matrix (for a survey on compound matrices and their relations to differential equations see [11]) and in the special case $n=3$, one has

$$
J^{[2]}=\left(\begin{array}{ccc}
J_{11}+J_{22} & J_{23} & -J_{13} \\
J_{32} & J_{11}+J_{33} & J_{12} \\
-J_{31} & J_{21} & J_{22}+J_{33}
\end{array}\right)
$$

Consider the Lozinskil measure $\mu$ of $B$ with respect to a vector norm $|\cdot|$ in $R^{N}, N=\left(\begin{array}{c}n \\ 2\end{array}\right)$, (see [12])

$$
\mu(B)=\lim _{h \rightarrow 0^{+}} \frac{\|I+h B\|-1}{h} .
$$

It is proved in [10] that, if $\left(H_{1}\right)$ and $\left(H_{2}\right)$ hold, condition

$$
q=\limsup _{t \rightarrow \infty} \sup _{x_{0} \in K} \frac{1}{t} \int_{0}^{t} \mu\left(B\left(x\left(s, x_{0}\right)\right)\right) \mathrm{d} s<0,
$$

guarantees that there are no orbits giving rise to a simple closed rectifiable curve in $D$ which is invariant for (23), that is, periodic orbits, homoclinic orbits, and heteroclinic cycles. In particular, condition (28) is proved to be a robust Bendixson criterion for (23). Besides, it is remarked that, under the assumptions $\left(H_{1}\right)$ and $\left(H_{1}\right),(28)$ also implies the local stability of $x^{*}$.

The analysis of the global stability of the endemic equilibrium may be usefully approached by means of the PoincareBendixson trichotomy. If the endemic equilibrium is globally asymptotically stable, then the disease will permanently be present in the population in case of infinitesimal initial prevalence. Here we will provide an analytical proof of global stability of $E$ by giving sufficient conditions. Global stability analysis for the endemic equilibrium will be performed 
through the approach due to Li and Muldowney. The instability of $E_{1}$ implies the uniform persistence; that is, there exists a constant $a>0$ such that any solution $(S(t), I(t), Z(t))$ with $(S(0), I(0), Z(0))$ in the orbit of the system satisfies

$$
\min \left\{\liminf _{t \rightarrow \infty} S(t), \operatorname{limin}_{t \rightarrow \infty} I(t), \liminf _{t \rightarrow \infty} Z(t)\right\}>a
$$

Consider the following assumptions:

$$
\begin{gathered}
a>\max \left\{\omega, \frac{k}{2}\right\}, \\
\mu_{1}+\gamma+\varepsilon>\max \left\{\frac{b a}{(\omega+a)^{2}}+\frac{\beta k}{(1+a \alpha)^{2}}, \frac{\beta k}{1+k \alpha}\right\}, \\
\frac{\beta a(2+a \alpha)}{(1+a \alpha)^{2}}>\frac{b}{\omega+a}, \\
\delta>\mu_{1}+\gamma+\varepsilon>0,
\end{gathered}
$$

$$
J=\left(\begin{array}{cccc}
r\left(1-\frac{S}{k}\right)-\frac{\beta I}{1+\alpha I}-\frac{r}{k} S & \frac{-\beta S}{(1+\alpha I)^{2}} & 0 \\
0 & \frac{\beta Z}{1+\alpha Z}-\left(\mu_{1}+\gamma+\varepsilon\right)-\frac{b \omega}{(\omega+I)^{2}} & \frac{\beta I}{(1+\alpha Z)^{2}} \\
\frac{1}{T} & 0 & -\frac{1}{T}
\end{array}\right)
$$

The second additive compound matrix $J^{[2]}$ of $J$ is

$$
J^{[2]}=\left(\begin{array}{ccc}
r\left(1-\frac{S}{k}\right)-\frac{\beta I}{1+\alpha I}-\frac{r}{k} S & \beta I \\
+\frac{\beta Z}{1+\alpha Z}-\left(\mu_{1}+\gamma+\varepsilon\right)-\frac{b \omega}{(\omega+I)^{2}} & r\left(1-\frac{S}{k}\right)-\frac{\beta I}{1+\alpha I}-\frac{r}{k} S-\frac{1}{T} & \frac{-\beta S}{1+\alpha S} \\
0 & 0 & \frac{\beta Z}{1+\alpha Z}-\frac{b \omega}{(\omega+I)^{2}} \\
-\frac{1}{T} & -\left(\mu_{1}+\gamma+\varepsilon\right)-\frac{1}{T}
\end{array}\right) .
$$

Consider the function $P=P(S, I, Z)=\operatorname{diag}\{S / I, S / I, S\}$.

Then

$$
\begin{gathered}
P^{-1}=\operatorname{diag}\left\{\frac{I}{S}, \frac{I}{S}, \frac{1}{S}\right\}, \\
P_{f}=\operatorname{diag}\left\{\frac{S^{\prime}}{I}-\frac{S}{I^{2}} I^{\prime}, \frac{S^{\prime}}{I}-\frac{S}{I^{2}} I^{\prime}, S^{\prime}\right\} .
\end{gathered}
$$

Lemma 6 (Li and Muldowney [10]). Assume that conditions $\left(H_{1}\right)$ and $\left(H_{2}\right)$ hold; then $x^{*}$ is globally asymptotically stable in $D$ provided that a function $P(x)$ and a Lozinskil measure $\mu$ exist such that (28) is satisfied.

Theorem 7. Under the assumption $\mathscr{R}_{0}>1, \overline{\mathscr{R}}_{0}<1+$ $k \alpha-(k / r) \beta,(29)$, and (30), the endemic equilibrium $E^{*}$ of the system (6) is globally asymptotically stable.

Proof. The Jacobian matrix at $E^{*}$ of system (6) is

$$
\begin{gathered}
\delta=\min \left\{\frac{2 r a}{k}+\frac{\beta a}{1+a \alpha}+\left(\mu_{1}+\gamma+\varepsilon\right)\right. \\
-\frac{\beta k}{(1+a \alpha)^{2}}-\frac{b a}{(\omega+a)^{2}}-r, \\
\frac{\beta a(2+a \alpha)}{(1+k \alpha)^{2}}+\frac{2 r a}{k}-r-\frac{b}{\omega+a}, \\
\left.2\left(\mu_{1}+\gamma+\varepsilon\right)-\frac{\beta k}{1+k \alpha}\right\} .
\end{gathered}
$$


Therefore

where

$$
B=P_{f} P^{-1}+P J^{[2]} P^{-1}=\left(\begin{array}{ll}
B_{11} & B_{12} \\
B_{21} & B_{22}
\end{array}\right),
$$

$$
\begin{gathered}
B_{11}=\frac{S^{\prime}}{S}-\frac{I^{\prime}}{I}+r\left(1-\frac{S}{k}\right)-\frac{\beta I}{1+\alpha I}-\frac{r}{k} S+\frac{\beta Z}{1+\alpha Z}-\left(\mu_{1}+\gamma+\varepsilon\right)-\frac{b \omega}{(\omega+I)^{2}}, \\
B_{12}=\left(\frac{\beta I}{(1+\alpha Z)^{2}}, 0\right), \quad B_{21}=\left(0,-\frac{1}{T}\right)^{T}, \\
B_{22}=\left(\begin{array}{c}
\frac{S^{\prime}}{S}-\frac{I^{\prime}}{I}+r-\frac{2 r S}{k}-\frac{\beta I}{1+\alpha I}-\frac{1}{T} \quad \frac{-\beta S}{(1+\alpha I)^{2}} \\
0 \quad \frac{S^{\prime}}{S}+\frac{\beta Z}{1+\alpha Z}-\left(\mu_{1}+\gamma+\varepsilon\right)-\frac{b \omega}{(\omega+I)^{2}}-\frac{1}{T}
\end{array}\right) .
\end{gathered}
$$

Consider the norm in $R^{3}$ as

$$
\|(u, v, \omega)\|=\max \{|u|,|v|+|\omega|\},
$$

where $(u, v, \omega)$ denotes vector $R^{3}$ and $\mu(B)$ denotes the Lozinskil measure with respect to the $L^{1}$ norm

$$
\mu(B) \leq \sup \left\{g_{1}, g_{2}\right\}
$$

where $g_{1}=\mu_{1}\left(B_{11}\right)+\left|B_{12}\right|$ and $g_{2}=\mu_{1}\left(B_{22}\right)+\left|B_{21}\right|$. Here $\left|B_{12}\right|$ and $\left|B_{21}\right|$ are matrix norms with respect to the $L^{1}$ vector norm and $\mu_{1}$ denotes the Lozinskil measure with respect to the $L^{1}$ norm.

Therefore

$$
\begin{aligned}
& \mu_{1}\left(B_{11}\right)=\frac{S^{\prime}}{S}-\frac{I^{\prime}}{I}+r\left(1-\frac{S}{k}\right) \\
& -\frac{\beta I}{1+\alpha I}-\frac{r}{k} S+\frac{\beta Z}{1+\alpha Z} \\
& -\left(\mu_{1}+\gamma+\varepsilon\right)-\frac{b \omega}{(\omega+I)^{2}}, \\
& \left|B_{12}\right|=\frac{\beta I}{(1+\alpha Z)^{2}}, \quad\left|B_{21}\right|=\frac{1}{T}, \\
& \mu_{1}\left(B_{22}\right)=\mu_{1}\left(B_{11}\right)+\left|B_{12}\right| \\
& =\frac{S^{\prime}}{S}-\frac{I^{\prime}}{I}-\frac{1}{T} \\
& +\max \left\{r-\frac{2 r S}{k}-\frac{\beta I}{1+\alpha I}, \frac{I^{\prime}}{I}+\frac{\beta Z}{1+\alpha Z}\right. \\
& \left.-\left(\mu_{1}+\gamma+\varepsilon\right)-\frac{b \omega}{(\omega+I)^{2}}\right\} \text {. }
\end{aligned}
$$

Therefore $g_{1}=\left(S^{\prime} / S\right)-\left(I^{\prime} / I\right)+r-(2 r S / k)-(\beta I /(1+\alpha I))+$ $\left(\beta I /(1+\alpha Z)^{2}\right)+(\beta Z /(1+\alpha Z))-\left(\mu_{1}+\gamma+\varepsilon\right)-\left(b \omega /(\omega+I)^{2}\right)$,

$$
\begin{aligned}
& g_{2}= \mu_{1}\left(B_{22}\right)+\left|B_{21}\right|=\frac{S^{\prime}}{S}-\frac{I^{\prime}}{I} \\
&+\max \left\{r-\frac{2 r S}{k}-\frac{\beta I}{1+\alpha I}, \frac{I^{\prime}}{I}+\frac{\beta Z}{1+\alpha Z}\right. \\
&\left.-\left(\mu_{1}+\gamma+\varepsilon\right)-\frac{b \omega}{(\omega+I)^{2}}\right\} .
\end{aligned}
$$

From the system $(6), I^{\prime} / I=(\beta Z /(1+\alpha Z))-\left(\mu_{1}+\gamma+\varepsilon\right)-$ $(b /(\omega+I))$; therefore

$$
\begin{aligned}
& g_{1}= \frac{S^{\prime}}{S}-\frac{\beta Z}{1+\alpha Z}+\left(\mu_{1}+\gamma+\varepsilon\right)+\frac{b}{\omega+I} \\
&+r-\frac{2 r S}{k}-\frac{\beta I}{1+\alpha I}+\frac{\beta I}{(1+\alpha Z)^{2}}+\frac{\beta Z}{1+\alpha Z} \\
&-\left(\mu_{1}+\gamma+\varepsilon\right)-\frac{b \omega}{(\omega+I)^{2}} \\
&= \frac{S^{\prime}}{S}+\left(\mu_{1}+\gamma+\varepsilon\right)+r-\frac{2 r S}{k} \\
&-\frac{\beta I}{1+\alpha I}+\frac{\beta I}{(1+\alpha Z)^{2}}-\left(\mu_{1}+\gamma+\varepsilon\right)+\frac{b I}{(\omega+I)^{2}}, \\
& g_{2}= \frac{S^{\prime}}{S}-\frac{\beta Z}{1+\alpha Z}+\left(\mu_{1}+\gamma+\varepsilon\right)+\frac{b}{\omega+I} \\
&+\max \left\{r-\frac{2 r S}{k}-\frac{\beta I}{1+\alpha I}, \frac{2 \beta Z}{1+\alpha Z}\right. \\
&\left.-2\left(\mu_{1}+\gamma+\varepsilon\right)-\frac{2 b \omega+b I}{(\omega+I)^{2}}\right\}
\end{aligned}
$$




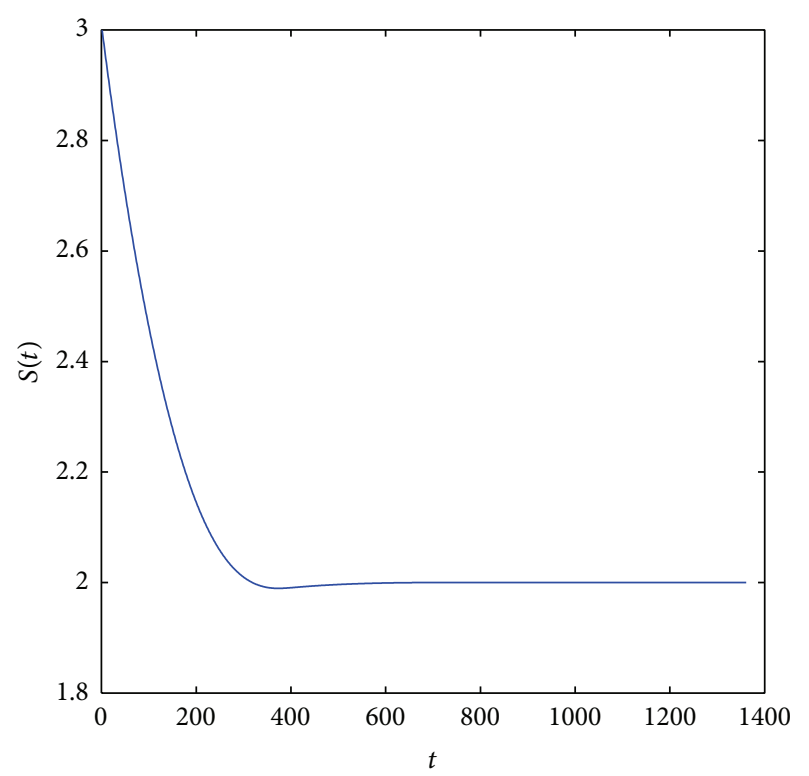

(a)

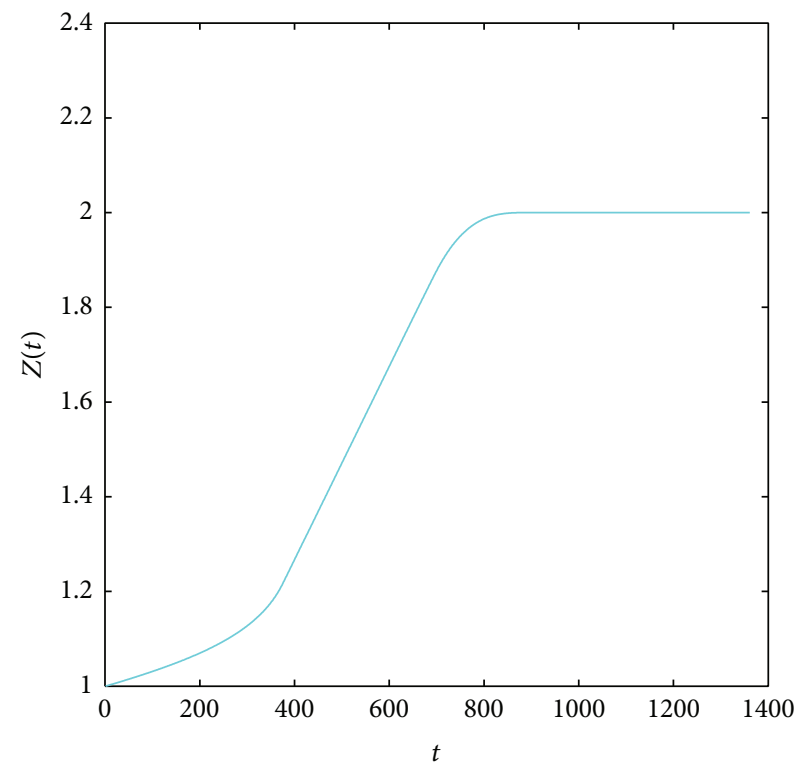

(c)

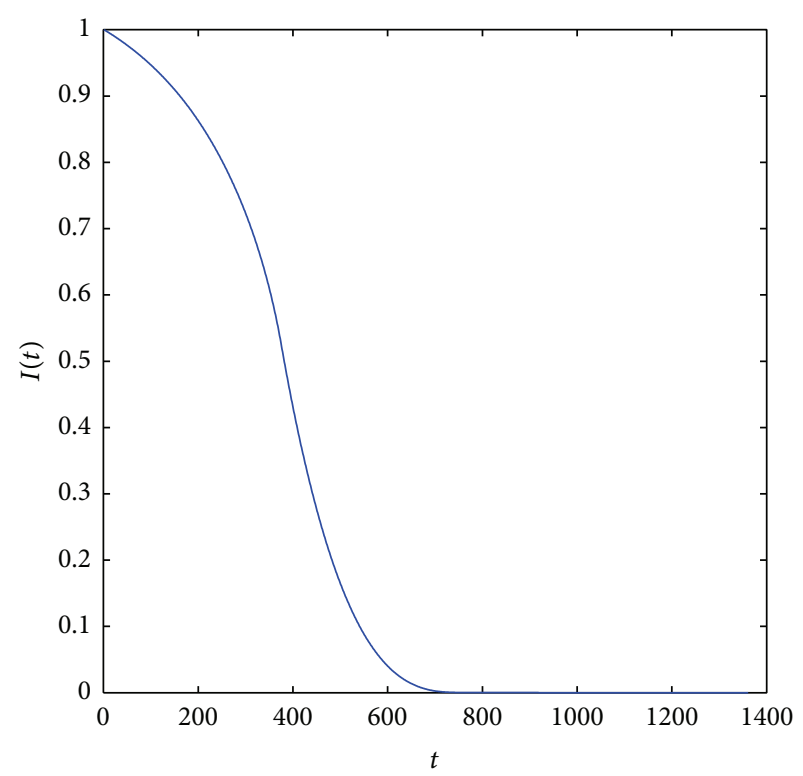

(b)

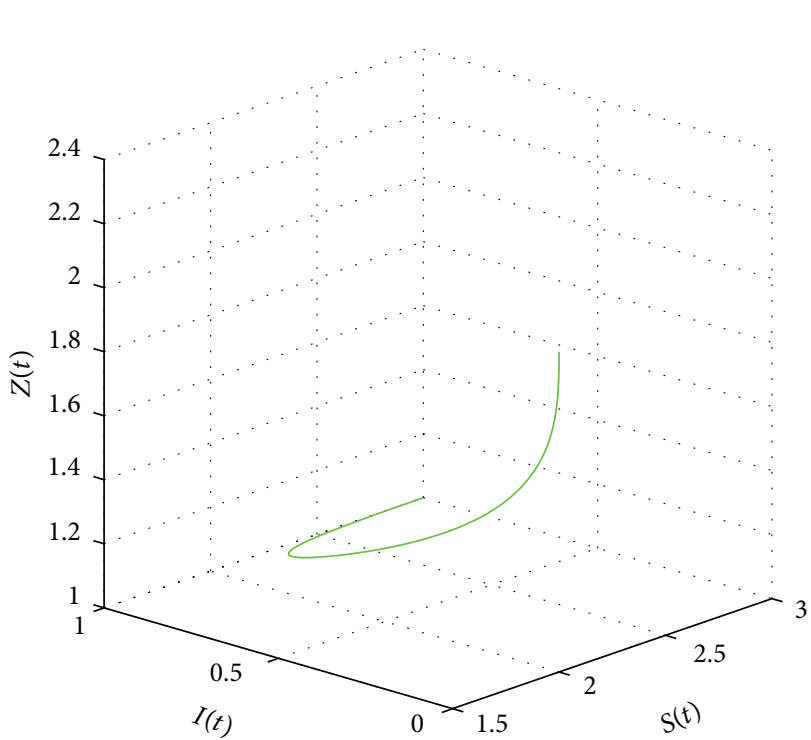

(d)

FIGURE 1: (a)-(d) showed that the equilibrium $E_{1}$ of system (6) with initial condition $S(0)=3 ; I(0)=1 ; Z(0)=1 ; R_{0}=0.1786<1 ;$ and $T=4$ is locally asymptotically stable.

$$
\begin{aligned}
&=\frac{S^{\prime}}{S}+\left(\mu_{1}+\gamma+\varepsilon\right) \\
&+\max \left\{r+\frac{b}{\omega+I}-\frac{2 r S}{k}-\frac{\beta I}{1+\alpha I}\right. \\
&-\frac{\beta Z}{1+\alpha Z}, \frac{\beta Z}{1+\alpha Z} \\
&\left.-2\left(\mu_{1}+\gamma+\varepsilon\right)-\frac{b \omega}{(\omega+I)^{2}}\right\} .
\end{aligned}
$$

Hence

$$
\begin{aligned}
\mu(B) \leq & \sup \left\{g_{1}, g_{2}\right\} \\
\leq & \frac{S^{\prime}}{S}+\left(\mu_{1}+\gamma+\varepsilon\right) \\
& +\max \left\{r-\frac{2 r S}{k}-\frac{\beta I}{1+\alpha I}+\frac{\beta I}{(1+\alpha Z)^{2}}\right. \\
& \quad-\left(\mu_{1}+\gamma+\varepsilon\right)+\frac{b I}{(\omega+I)^{2}},
\end{aligned}
$$




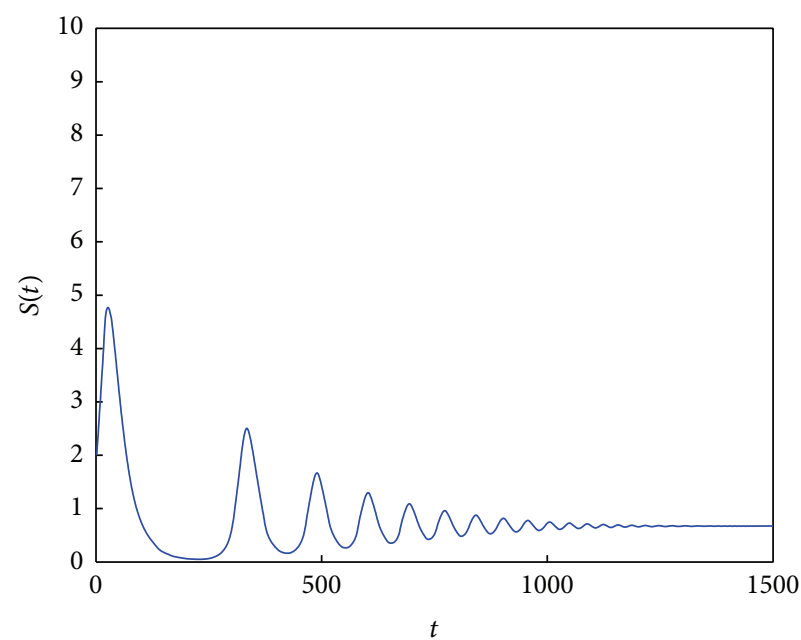

(a)

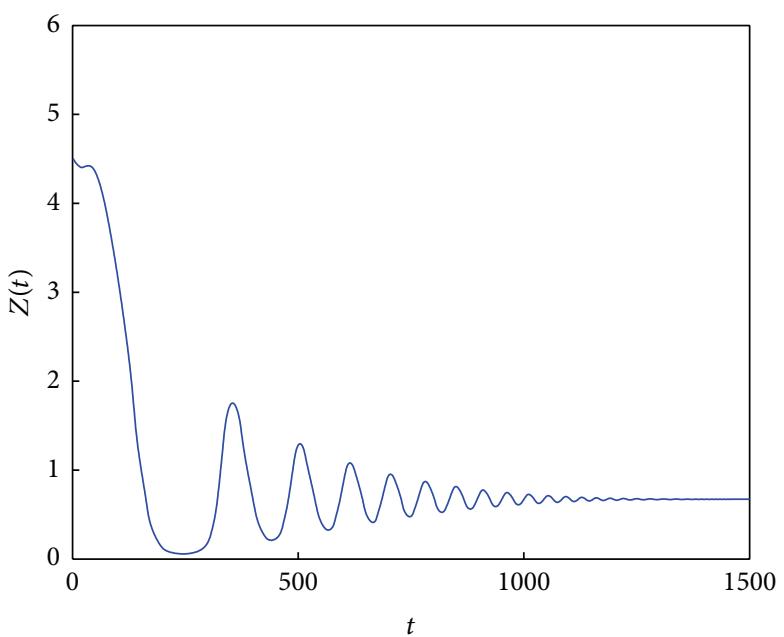

(c)

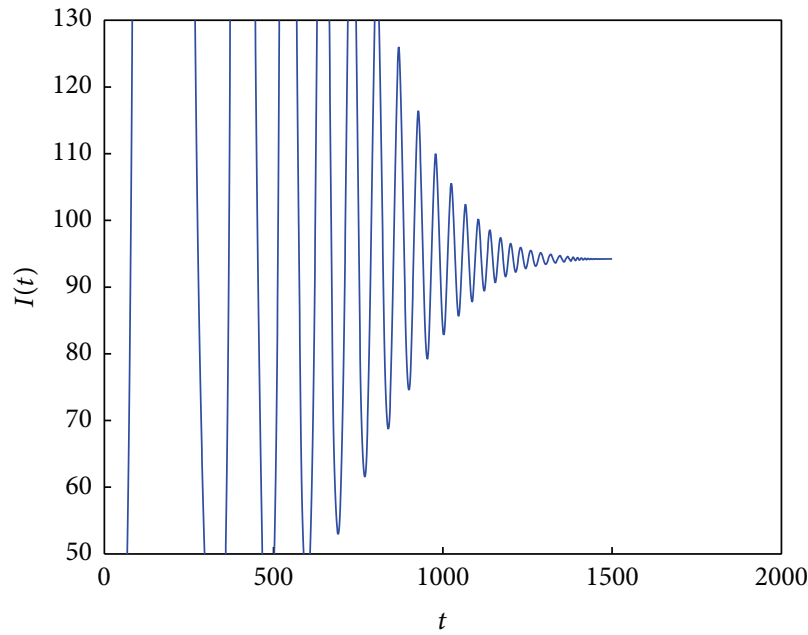

(b)

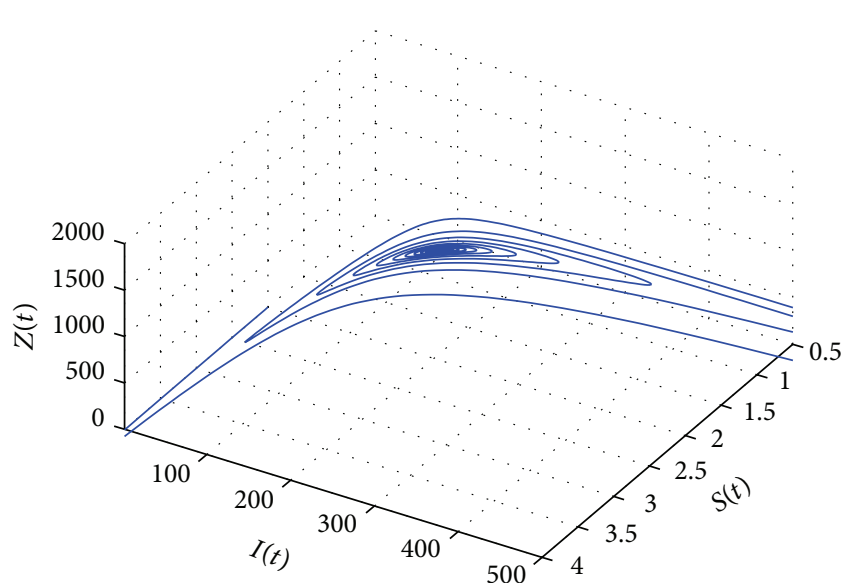

(d)

FIGURE 2: (a)-(d) showed that equilibrium $E^{*}$ of system (6) with initial condition $S(0)=2 ; I(0)=2 ; Z(0)=4.5 ; R_{0}=3.6692>1$; and $T=10<T_{*}$ is locally asymptotically stable.

$$
\begin{aligned}
& r+\frac{b}{\omega+I}-\frac{2 r S}{k}-\frac{\beta I}{(1+\alpha S)^{2}} \\
& -\frac{\beta Z}{1+\alpha Z}, \frac{\beta Z}{1+\alpha Z} \\
& \left.-2\left(\mu_{1}+\gamma+\varepsilon\right)-\frac{b \omega}{(\omega+I)^{2}}\right\} .
\end{aligned}
$$

That is,

$$
\begin{aligned}
& \mu(B) \leq \frac{S^{\prime}}{S}+\left(\mu_{1}+\gamma+\varepsilon\right) \\
&+\max \left\{r-\frac{2 r a}{k}-\frac{\beta a}{1+a \alpha}+\frac{\beta k}{(1+a \alpha)^{2}}\right. \\
&-\left(\mu_{1}+\gamma+\varepsilon\right)+\frac{b a}{(\omega+a)^{2}}
\end{aligned}
$$

$$
\begin{gathered}
r+\frac{b}{\omega+a}-\frac{2 r a}{k}-\frac{\beta a(2+a \alpha)}{(1+k \alpha)^{2}}, \\
\left.\frac{\beta k}{1+\alpha k}-2\left(\mu_{1}+\gamma+\varepsilon\right)\right\} \\
\leq \frac{S^{\prime}}{S}+\left(\mu_{1}+\gamma+\varepsilon\right) \\
-\min \left\{\frac{2 r a}{k}+\frac{\beta a}{1+a \alpha}+\left(\mu_{1}+\gamma+\varepsilon\right)\right. \\
-\frac{\beta k}{(1+a \alpha)^{2}}-\frac{b a}{(\omega+a)^{2}}-r \\
\frac{\beta a(2+a \alpha)}{(1+k \alpha)^{2}}+\frac{2 r a}{k}-r \\
-\frac{b}{\omega+a}, 2\left(\mu_{1}+\gamma+\varepsilon\right)
\end{gathered}
$$




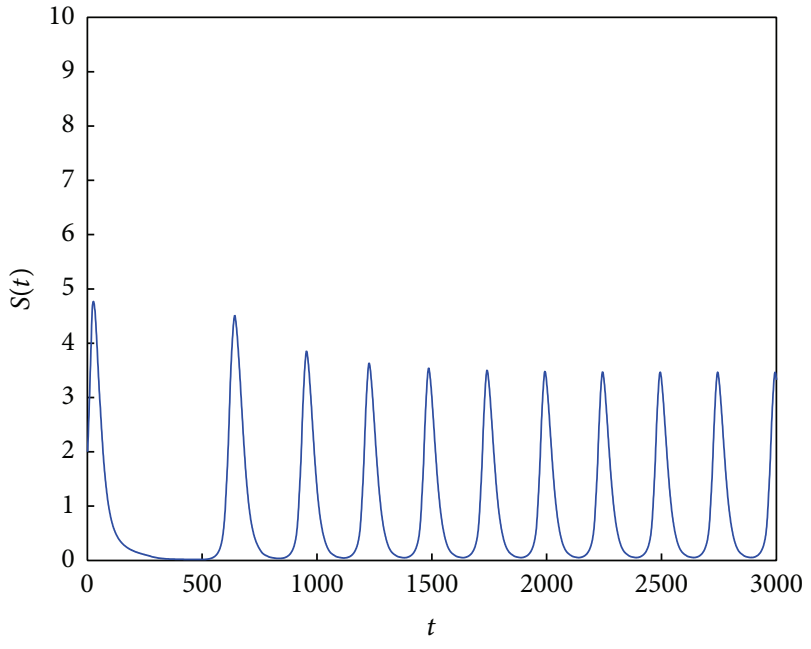

(a)

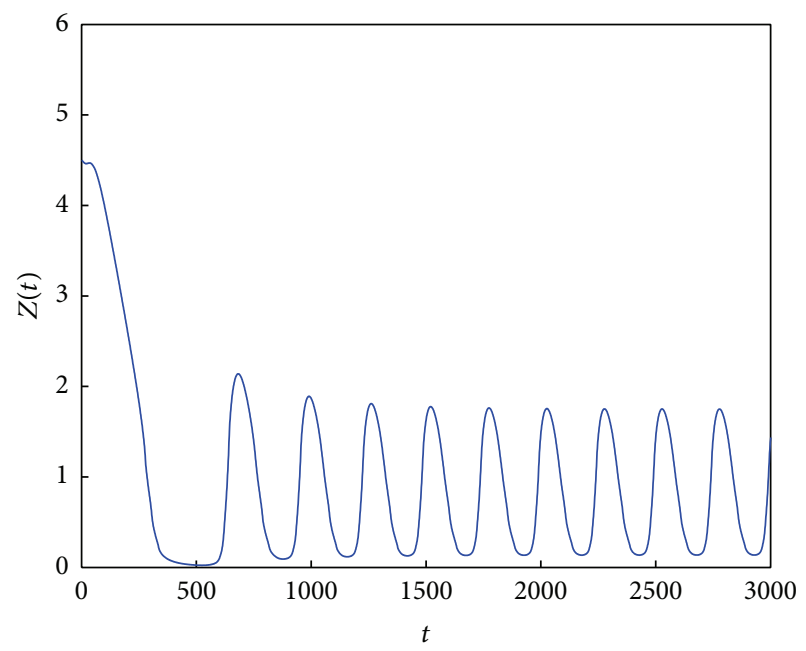

(c)

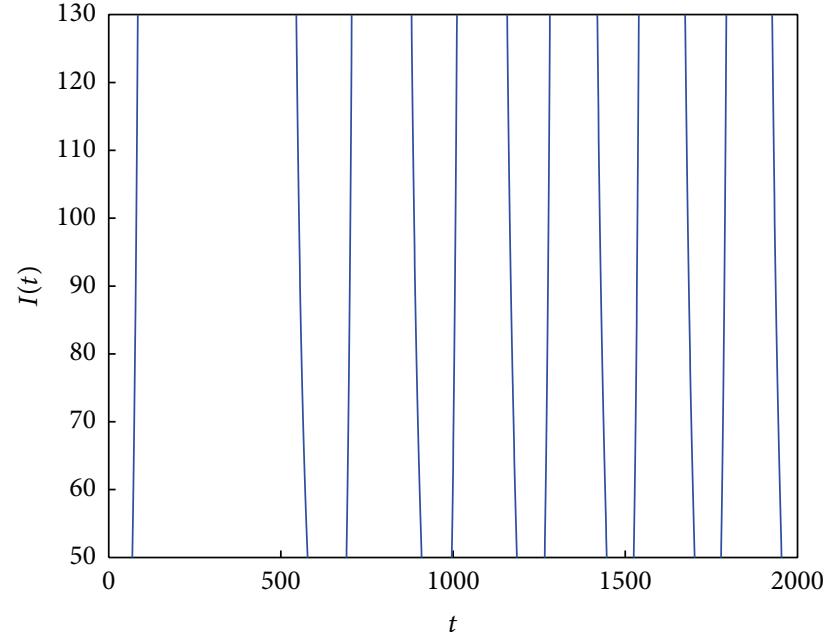

(b)

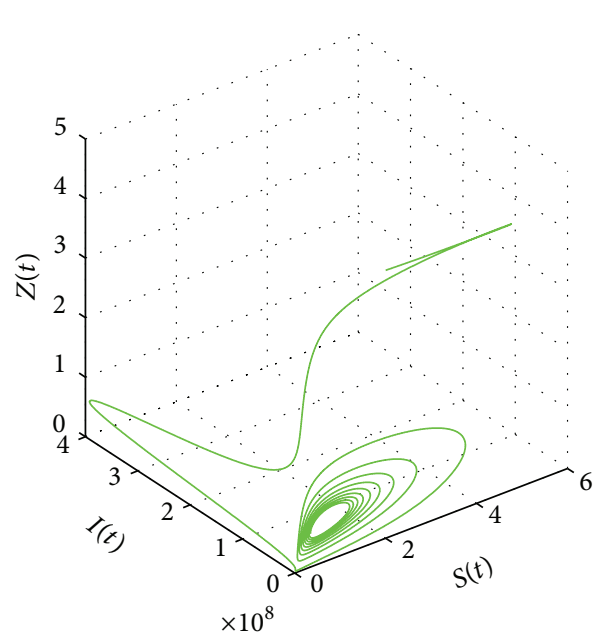

(d)

Figure 3: (a)-(d) showed that equilibrium $E^{*}$ of system (6) with initial condition $S(0)=2 ; I(0)=2 ; Z(0)=4.5 ; R_{0}=3.6692>1 ;$ and $T=25>T_{*}$ is unstable.

$$
\begin{array}{r}
\left.-\frac{\beta k}{1+k \alpha}\right\} \\
\leq \frac{S^{\prime}}{S}+\left(\mu_{1}+\gamma+\varepsilon\right)-\delta .
\end{array}
$$

Hence

$$
\mu(B) \leq \frac{S^{\prime}}{S}-\left(\delta-\mu_{1}-\gamma-\varepsilon\right) .
$$

By integrating both sides at the same time, we obtain

$$
\begin{gathered}
\frac{1}{t} \int_{0}^{t} \mu(B) \mathrm{d} s \leq \frac{1}{t} \ln \frac{S(t)}{S(0)}-\left(\delta-\mu_{1}-\gamma-\varepsilon\right), \\
\limsup _{t \rightarrow \infty} \sup \frac{1}{t} \int_{0}^{t} \mu(B) \mathrm{d} s<-\left(\delta-\mu_{1}-\gamma-\varepsilon\right)<0 .
\end{gathered}
$$

The proof is completed by Lemma 6 and (30).

\section{Numerical Simulations}

To demonstrate the theoretical results obtained in this paper, we will give some numerical simulations. We consider the hypothetical set of parameter values as follows.

(1) Consider $k=2 ; \beta=0.03 ; \mu_{1}=0.1 ; \mu_{2}=0.2$; $r=3 ; b=2 ; \alpha=0.001 ; \gamma=0.05 ; T=4$; $\varepsilon=0.02 ; \omega=12$. By directly computing, we obtain $R_{0}=0.1786<1$. According to Theorem 3, we know the free disease equilibrium of system (6) is locally asymptotically stable for this case (see Figures 1(a)$1(\mathrm{c})$ and $1(\mathrm{~d})$ ).

(2) Consider $k=7 ; \beta=0.3 ; \mu_{1}=0.1 ; \mu_{2}=0.2$; $r=3 ; b=2 ; \alpha=0.1 ; \gamma=0.05 ; T=10$; $\varepsilon=0.02 ; \omega=12$. Through calculation, we know $R_{0}=3.6692>1, T<T_{*}$. According to Theorem 7, we know the positive equilibrium of system (6) is locally 
asymptotically stable for this case (see Figures 2(a)$2(\mathrm{c})$ and $2(\mathrm{~d}))$.

(3) Consider $k=7 ; \beta=0.3 ; \mu_{1}=0.1 ; \mu_{2}=0.2 ; r=3$; $b=2 ; \alpha=0.1 ; \gamma=0.05 ; T=25 ; \varepsilon=0.02 ; \omega=12$. Through calculation, we know $R_{0}=3.6692>1, T>$ $T_{*}$. According to Theorem 5, we know the positive equilibrium of system (6) is unstable for this case (see Figures 3(a)-3(c) and 3(d)).

\section{Conclusion}

In this paper, the stability of the SIR epidemic model with information variable and limited medical resources has been revisited. By analyzing the model, we have found the diseasefree equilibria $E_{0}$ and $E_{1}$ exist when the basic reproduction ratio $\mathscr{R}_{0}<1$. At the same time we have proved the local asymptotic stability of the disease-free equilibrium. The conclusion reveals that the disease dies out, when $\mathscr{R}_{0}>1$; then disease becomes endemic. $\mathscr{R}_{0}$ changes the stability of the disease-free equilibrium and delay parameter $T$ and $\omega$ change the stability of the endemic equilibrium. It is shown that the disease-free equilibrium is unstable and the unique endemic equilibrium is globally asymptotically stable under some conditions. Lastly, a numerical simulation provided that, when $\mathscr{R}_{0}$ is less than 1 , the disease-free equilibrium is stable and while $\mathscr{R}_{0}$ is more than 1 , the disease-free equilibrium is unstable; that is, the endemic equilibrium exists (see Figure 1). We found that if $T<T_{*}$, the equilibrium $E^{*}$ is globally asymptotically stable (see Figure 2 ).

If $T>T_{*}$, it is concluded that the instability of the equilibrium $E^{*}$ has not been studied. It is worthwhile for us to study this case in the future work from the theorematic idea. Here we only illustrate the equilibrium $E^{*}$ is unstable if $T>T_{*}$ by use of the numerical simulation (see Figure 3 ).

\section{Conflict of Interests}

The authors declare that there is no conflict of interests regarding the publication of this paper.

\section{Acknowledgments}

The authors would like to thank the anonymous referees for their careful reading of the original paper and their many valuable comments and suggestions that greatly improve the presentation of this work. This work is supported by Natural Science of Shanxi Province (2013011002-2).

\section{References}

[1] L. Cai, S. Guo, X. Li, and M. Ghosh, "Global dynamics of a dengue epidemic mathematical model," Chaos, Solitons \& Fractals, vol. 42, no. 4, pp. 2297-2304, 2009.

[2] J.-Z. Zhang, Z. Jin, Q.-X. Liu, and Z.-Y. Zhang, "Analysis of a delayed SIR model with nonlinear incidence rate," Discrete Dynamics in Nature and Society, vol. 2008, Article ID 636153, 16 pages, 2008.
[3] B. Buonomo, A. d'Onofrio, and D. Lacitignola, "Global stability of an SIR epidemic model with information dependent vaccination," Mathematical Biosciences, vol. 216, no. 1, pp. 9-16, 2008.

[4] A. d'Onofrio, P. Manfredi, and E. Salinelli, "Vaccinating behaviour, information, and the dynamics of SIR vaccine preventable diseases," Theoretical Population Biology, vol. 71, no. 3, pp. 301-317, 2007.

[5] A. d'Onofrio, P. Manfredi, and E. Salinelli, "Bifurcation thresholds in an SIR model with information-dependent vaccination," Mathematical Modelling of Natural Phenomena, vol. 2, no. 1, pp. 26-43, 2007.

[6] X. Zhang and X. N. Liu, "Backward bifurcation of an epidemic model with saturated treatment function," Journal of Mathematical Analysis and Applications, vol. 348, no. 1, pp. 433-443, 2008.

[7] L. Zhou and M. Fan, "Dynamics of an SIR epidemic model with limited medical resources revisited," Nonlinear Analysis: Real World Applications, vol. 13, no. 1, pp. 312-324, 2012.

[8] J. Cui, X. Mu, and $\mathrm{H}$. Wan, "Saturation recovery leads to multiple endemic equilibria and backward bifurcation," Journal of Theoretical Biology, vol. 254, no. 2, pp. 275-283, 2008.

[9] J. Guckenheimer and P. Holmes, Nonlinear Oscillations, Dynamical Systems, and Bifurcations of Vector Fields, Springer, New York, NY, USA, 1983.

[10] M. Y. Li and J. S. Muldowney, "A geometric approach to globalstability problems," SIAM Journal on Mathematical Analysis, vol. 27, no. 4, pp. 1070-1083, 1996.

[11] R. H. Martin Jr., "Logarithmic norms and projections applied to linear differential systems," Journal of Mathematical Analysis and Applications, vol. 45, no. 2, pp. 432-454, 1974.

[12] J. S. Muldowney, "Compound matrices and ordinary differential equations," Rocky Mountain Journal of Mathematics, vol. 20, no. 4, pp. 857-872, 1990. 


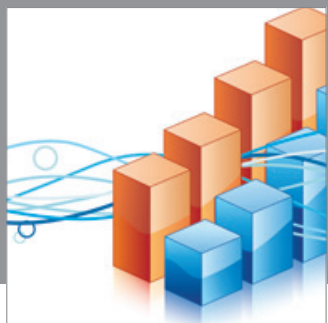

Advances in

Operations Research

mansans

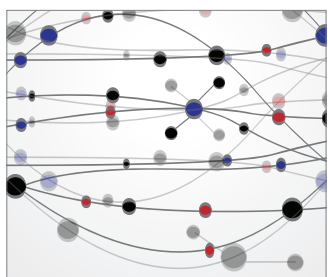

The Scientific World Journal
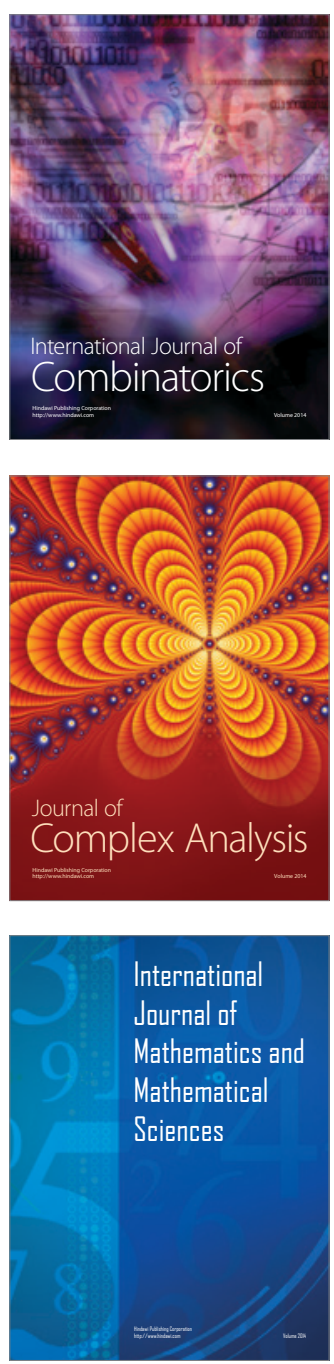
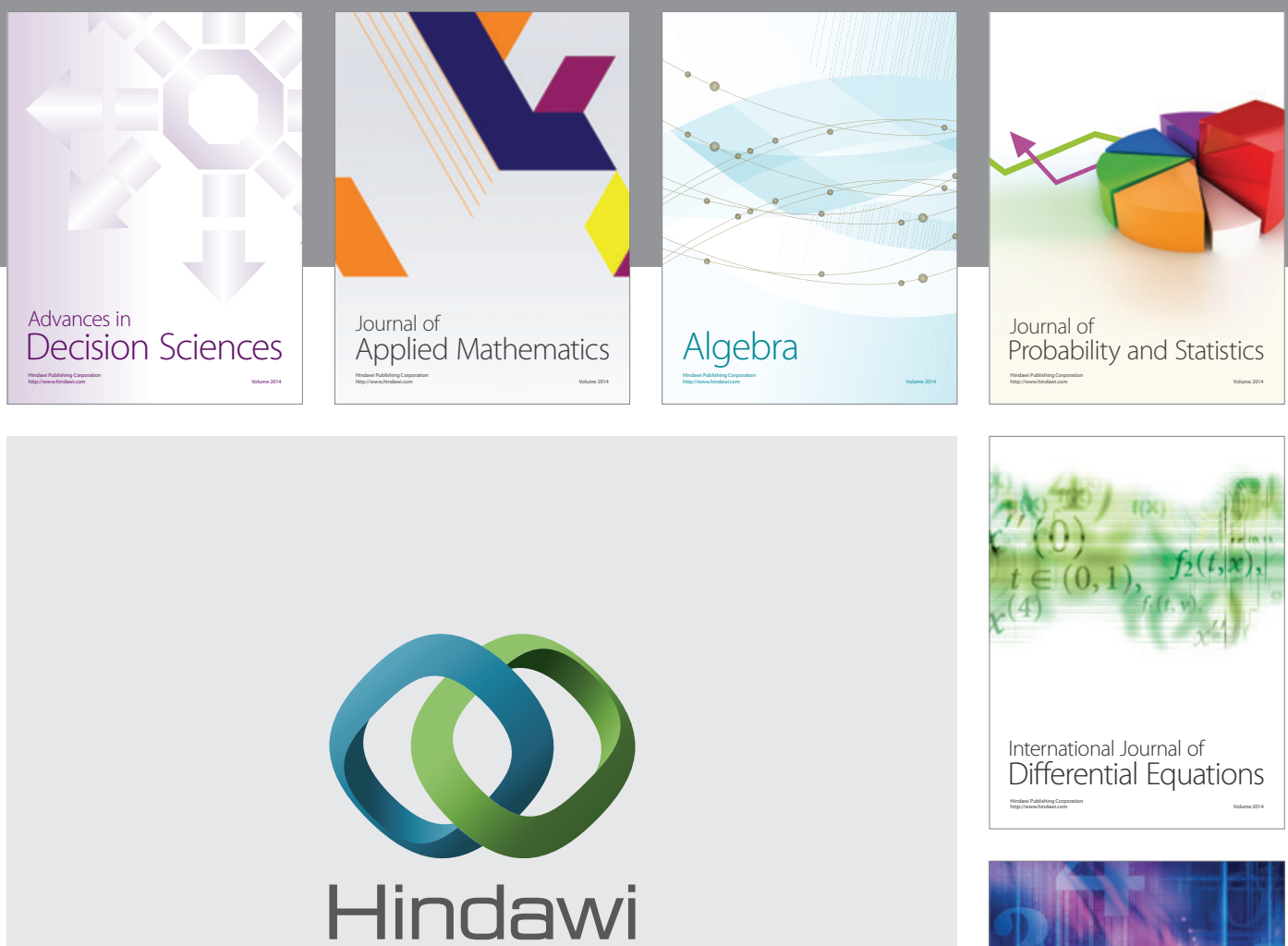

Submit your manuscripts at http://www.hindawi.com
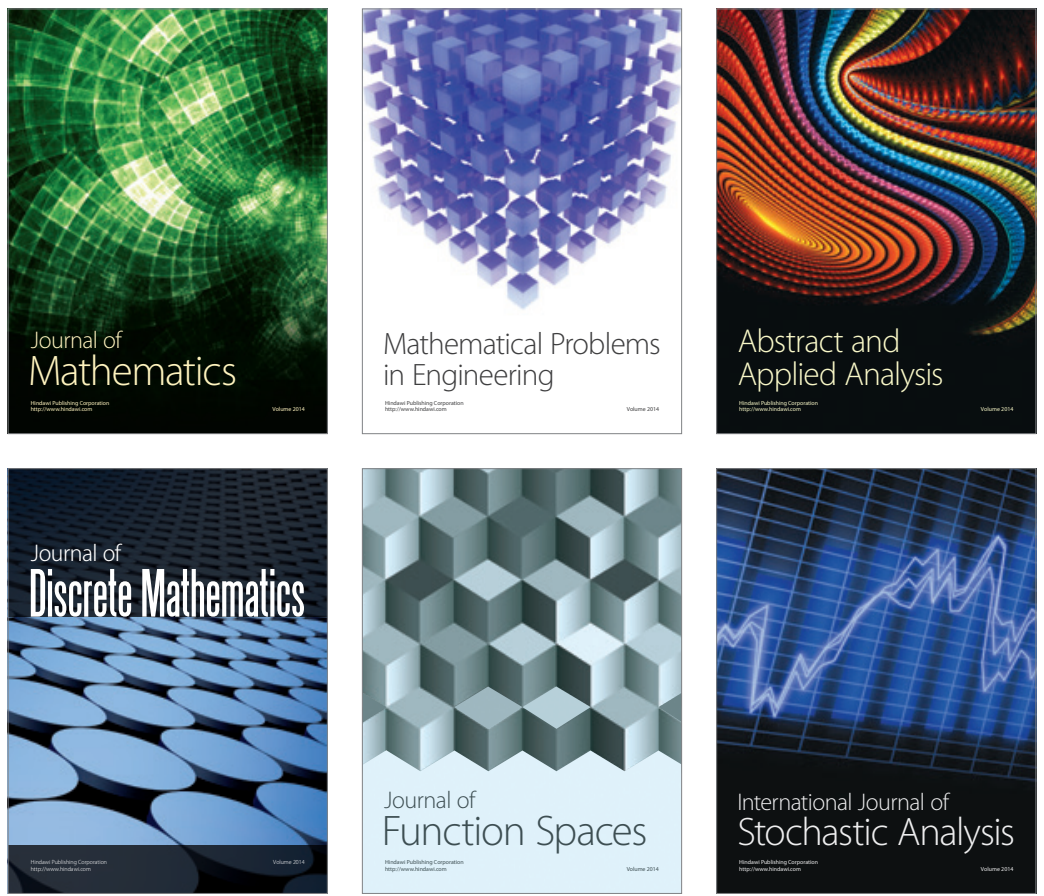

Journal of

Function Spaces

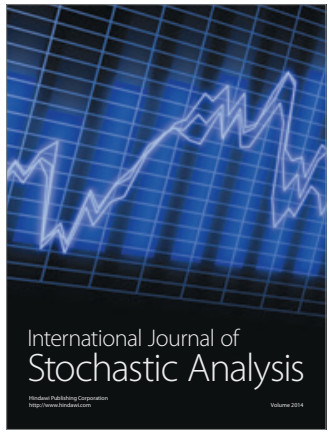

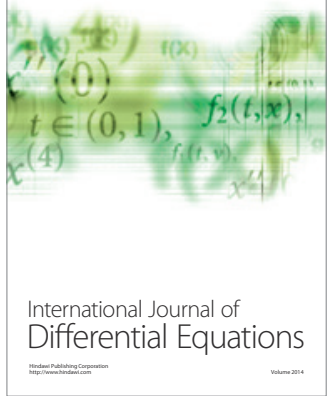
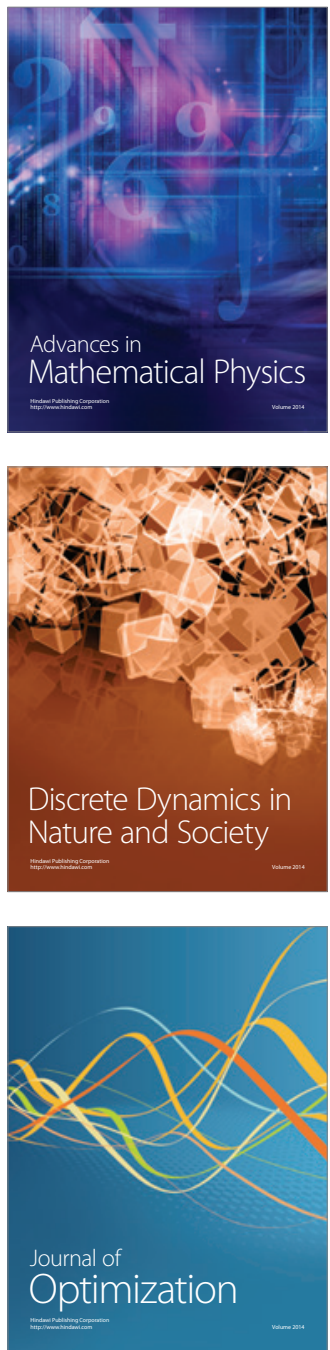